K.H. Kwon · D.W. Lee · F. Marcellán · S.B. Park

\title{
On Kernel polynomials and self-perturbation of orthogonal polynomials
}

\begin{abstract}
Given an orthogonal polynomial system $\left\{Q_{n}(x)\right\}_{n=0}^{\infty}$, def ne another polynomial system by

$$
P_{n}(x)=Q_{n}(x)-\alpha_{n} Q_{n-t}(x), \quad n \geq 0,
$$

where $\alpha_{n}$ are complex numbers and $t$ is a positive integer. We fi d conditions for $\left\{P_{n}(x)\right\}_{n=0}^{\infty}$ to be an orthogonal polynomial system. When $t=1$ and $\alpha_{1} \neq 0$, it turns out that $\left\{Q_{n}(x)\right\}_{n=0}^{\infty}$ must be kernel polynomials for $\left\{P_{n}(x)\right\}_{n=0}^{\infty}$ for which we study, in detail, the location of zeros and semi-classical character.
\end{abstract}

Mathematics Subject Classification (2000). 42C05

Key words. kernel polynomials - orthogonal polynomials

\section{Introduction}

For an orthogonal polynomial system $\left\{P_{n}(x)\right\}_{n=0}^{\infty}$ and a complex number $\lambda$ with $P_{n}(\lambda) \neq 0, n \geq 1$, its kernel polynomial system $\left\{P_{n}^{*}(\lambda ; x)\right\}_{n=0}^{\infty}$ can be introduced (cf. [3] and [3, p.35]) and $(x-\lambda) P_{n}^{*}(\lambda ; x)$ can be expressed as a linear combination of $P_{n}(x)$ and $P_{n-1}(x)$. Conversely, $P_{n}(x)$ can be expressed as a linear combination of $P_{n}^{*}(\lambda ; x)$ and $P_{n-1}^{*}(\lambda ; x)$ (cf. (2.5)). In fact, this last property characterizes kernel polynomial systems: an OPS $\left\{Q_{n}(x)\right\}_{n=0}^{\infty}$ is a kernel polynomial system for some other orthogonal polynomial system if and only if

$$
Q_{n}(x)-\alpha_{n} Q_{n-1}(x), \quad n \geq 0
$$

becomes an orthogonal polynomial system for some complex numbers $\alpha_{n}$, with $\alpha_{1} \neq 0$ (cf. Theorem 3.2). We may view (1.1) as a self-perturbation of orthogonal

K.H. Kwon: Division of Applied Mathematics, KAIST, Taejon 305-701, Korea, e-mail: khkwon@jacobi.kaist.ac.kr

D.W. Lee: Dept. of Math., Teachers College, Kyungpook National University, Taegu 702701, Korea, e-mail: dwlee@gauss . knu.ac.kr

F. Marcellán: Dept. de Matematicas, Univ. Carlos III, Avenida Universidad 30, LeganésMadrid, Spain, e-mail: pacomarceing . uc3m.es

S.B. Park: Dept. of Mathematics, Korean Military Academy, Seoul 139-799, Korea 
polynomials $\left\{Q_{n}(x)\right\}_{n=0}^{\infty}$. More generally, we may ask: for a fi ed integer $t \geq 1$, given an orthogonal polynomial system $\left\{Q_{n}(x)\right\}_{n=0}^{\infty}$, when is the polynomial system

$$
Q_{n}(x)-\alpha_{n} Q_{n-t}(x), \quad(n \geq 0)
$$

also an orthogonal polynomial system? Here, $\alpha_{n}$ are complex numbers. Geronimus [5] posed and solves completely the case $t=1$. Later on Marcellán and Petronilho [12] stated the connection with the concept of coherent pairs of orthogonal polynomials. See also [2] for a similar problem.

In this work, we frst study kernel polynomials (i.e., the case when $t=1$ ) in some more detail, including their zero distribution and semi-classical character and then $\mathrm{f}$ nd necessary and suff cient conditions for polynomials def ned by (1.2) for $t \geq 2$ to be orthogonal polynomials.

\section{Preliminaries}

Let $\mathcal{P}$ be the space of all polynomials in one variable with complex coeff cients and $\operatorname{deg}(\phi)$ the degree of $\phi(x)$ in $\mathcal{P}$ with the convention that $\operatorname{deg}(0)=-1$. By a polynomial system (PS), we mean a sequence of polynomials $\left\{P_{n}(x)\right\}_{n=0}^{\infty}$ with $\operatorname{deg}\left(P_{n}\right)=n, n \geq 0$.

We call any linear functional $\sigma$ on $\mathcal{P}$ a moment functional and denote its action on a polynomial $\phi(x)$ by $\langle\sigma, \phi\rangle$. We say that a moment functional $\sigma$ is quasi-defi ite (respectively, positive-defi ite) if its moments $\sigma_{n}:=\left\langle\sigma, x^{n}\right\rangle$ satisfy the Hamburger condition

$$
\Delta_{n}(\sigma):=\operatorname{det}\left[\sigma_{i+j}\right]_{i, j=0}^{n} \neq 0
$$

(respectively, all $\sigma_{n}$ are real and $\left.\Delta_{n}(\sigma)>0\right), n=0,1, \ldots$

Definition 2.1. A PS $\left\{P_{n}(x)\right\}_{n=0}^{\infty}$ is called an orthogonal polynomial system (OPS) (respectively, a positive-definite OPS) if there is a moment functional $\sigma$ such that

$$
\left\langle\sigma, P_{m} P_{n}\right\rangle=K_{n} \delta_{m n}, \quad m, n=0,1, \ldots,
$$

where $K_{n} \neq 0$ (respectively, $K_{n}>0$ ), $n \geq 0$. In this case, we call $\left\{P_{n}(x)\right\}_{n=0}^{\infty}$ an OPS (respectively, a positive-definite OPS) relative to $\sigma$.

It is well known (see Theorem 3.1, Chapter 1 in [4]) that a moment functional $\sigma$ is quasi-defi ite if and only if there is an OPS $\left\{P_{n}(x)\right\}_{n=0}^{\infty}$ relative to $\sigma$. Moreover, in this case, each $\left\{P_{n}(x)\right\}_{n=0}^{\infty}$ is uniquely determined by $\sigma$ up to a non-zero constant factor. In particular, if each $P_{n}(x)$ is monic, we call $\left\{P_{n}(x)\right\}_{n=0}^{\infty}$ a monic OPS (MOPS) relative to $\sigma$. It's also well known that if $\left\{P_{n}(x)\right\}_{n=0}^{\infty}$ is an MOPS relative to a positive-defi ite moment functional $\sigma$, then each $P_{n}(x), n \geq 1$, has $n$ real simple zeros, which interlace with zeros of $P_{n+1}(x)$. We then let $[\xi, \eta](-\infty \leq \xi<\eta \leq \infty)$ be the true interval of orthogonality for $\left\{P_{n}(x)\right\}_{n=0}^{\infty}$ or $\sigma$, which is the smallest closed interval containing all zeros of $P_{n}(x), n \geq 1$. 
Due to Favard's theorem, a monic PS $\left\{P_{n}(x)\right\}_{n=0}^{\infty}$ is an OPS (respectively, a positive-defi ite OPS) if and only if $\left\{P_{n}(x)\right\}_{n=0}^{\infty}$ satisfie a three-term recurrence relation:

$$
P_{n+1}(x)=\left(x-b_{n}\right) P_{n}(x)-c_{n} P_{n-1}(x), \quad n \geq 0, \quad\left(P_{-1}=0\right),
$$

where $\left\{b_{n}\right\}_{n=0}^{\infty}$ and $\left\{c_{n}\right\}_{n=0}^{\infty}$ are complex numbers with $c_{n} \neq 0, n \geq 1$ (respectively, $b_{n}, n \geq 0$, are real and $\left.c_{n}>0, n \geq 1\right)$. For an MOPS $\left\{P_{n}(x)\right\}_{n=0}^{\infty}$ relative to $\sigma$ satisfying (2.1), we let $K_{n}(x, y),\left\{P_{n}^{(1)}(x)\right\}_{n=0}^{\infty}$, and $\left\{P_{n}(c ; x)\right\}_{n=0}^{\infty}$ be the $n$-th kernel polynomial, the associated MOPS of the frst kind, and the monic co-recursive OPS, respectively define by

$$
\begin{gathered}
K_{n}(x, y)=\sum_{k=0}^{n} \frac{P_{k}(x) P_{k}(y)}{\left\langle\sigma, P_{k}^{2}\right\rangle}, \quad n \geq 0 \\
P_{n+1}^{(1)}(x)=\left(x-b_{n+1}\right) P_{n}^{(1)}(x)-c_{n+1} P_{n-1}^{(1)}(x), \quad n \geq 0 \quad\left(P_{-1}^{(1)}(x)=0\right) ;
\end{gathered}
$$

and

$$
P_{n+1}(c ; x)=\left(x-b_{n}\right) P_{n}(c ; x)-c_{n} P_{n-1}(c ; x), \quad n \geq 1,
$$

where $P_{0}(c ; x)=0, P_{1}(c ; x)=P_{1}(x)-c$, and $c$ is a complex number.

For a moment functional $\sigma$, a polynomial $\pi(x)$, and a complex number $\lambda$, we let $\sigma^{\prime}, \pi \sigma$, and $(x-\lambda)^{-1} \sigma$ be the moment functionals def ned by

$$
\left\langle\sigma^{\prime}, P\right\rangle=-\left\langle\sigma, P^{\prime}\right\rangle, \quad\langle\pi \sigma, P\rangle=\langle\sigma, \pi P\rangle, \quad P \in \mathcal{P},
$$

and

$$
\left\langle(x-\lambda)^{-1} \sigma, P\right\rangle=\left\langle\sigma, \frac{P(x)-P(\lambda)}{x-\lambda}\right\rangle, \quad P \in \mathcal{P} .
$$

Definition 2.2 (Maroni [13]). A moment functional $\sigma$ is called semi-classical if $\sigma$ is quasi-definite and satisfies a Pearson-type functional equation

$$
(\alpha(x) \sigma)^{\prime}-\beta(x) \sigma=0
$$

for some polynomials $\alpha(x)$ and $\beta(x)$ with $\operatorname{deg}(\alpha) \geq 0$ and $\operatorname{deg}(\beta) \geq 1$.

For a semi-classical moment functional $\sigma$, we call

$$
s:=\min \max (\operatorname{deg}(\alpha)-2, \operatorname{deg}(\beta)-1)
$$

the class number of $\sigma$, where the minimum is taken over all pairs $(\alpha, \beta) \neq(0,0)$ of polynomials satisfying (2.2). We call an OPS relative to a semi-classical moment functional $\sigma$ of class $s$ a semi-classical OPS (SCOPS) of class $s$.

Next, we state two lemmas, which will be needed later.

Lemma 2.3. Let $\left\{P_{n}(x)\right\}_{n=0}^{\infty}$ be an OPS relative to a quasi-definite moment functional $\sigma$. Then for any moment functional $\tau,\left\langle\tau, P_{n}\right\rangle=0, n \geq k+1$ for some integer $k \geq 0$ if and only if $\tau=\phi \sigma$ for some polynomial $\phi(x)$ of degree $\leq k$. 
Proof. See Lemma 2.2 in [9].

Lemma 2.4. Let $\sigma$ and $\tau$ be moment functionals and $\lambda$ a complex number. Then $(x-\lambda) \sigma=\tau$ if and only if

$$
\sigma=(x-\lambda)^{-1} \tau+\sigma_{0} \delta(x-\lambda) .
$$

Proof. It is straightforward since the action of the moment functionals of both sides on $(x-\lambda)^{n}, n \geq 0$, coincides.

We now recall a few well-known facts on kernel polynomials (cf. [4]).

Proposition 2.5. Let $\left\{P_{n}(x)\right\}_{n=0}^{\infty}$ be an MOPS relative to $\sigma$. Then for a complex number $\lambda,(x-\lambda) \sigma$ is also quasi-definite if and only if $P_{n}(\lambda) \neq 0, n \geq 0$. In this case, the MOPS relative to $(x-\lambda) \sigma$ is given by

$$
\begin{aligned}
P_{n}^{*}(\lambda ; x) & =\frac{1}{P_{n}(\lambda)} \cdot \frac{P_{n+1}(x) P_{n}(\lambda)-P_{n+1}(\lambda) P_{n}(x)}{x-\lambda} \\
& =\frac{\left\langle\sigma, P_{n}^{2}\right\rangle}{P_{n}(\lambda)} K_{n}(x, \lambda), \quad n \geq 0,
\end{aligned}
$$

where

$$
K_{n}(x ; \lambda):=\sum_{k=0}^{n} \frac{P_{k}(x) P_{k}(\lambda)}{\left\langle\sigma, P_{k}^{2}\right\rangle}
$$

is the n-th kernel polynomial for $\left\{P_{n}(x)\right\}_{n=0}^{\infty}$ and

$$
\left\langle(x-\lambda) \sigma, P_{n}^{*}(\lambda ; x)^{2}\right\rangle=-\frac{P_{n+1}(\lambda)}{P_{n}(\lambda)}\left\langle\sigma, P_{n}^{2}\right\rangle, \quad n \geq 0 .
$$

Moreover, if $\sigma$ is positive-definite and $[\xi, \eta]$ is the true interval of orthogonality for $\sigma$, then the following are all equivalent:

(i) $(x-\lambda) \sigma$ is positive-definite;

(ii) $\operatorname{sgn} P_{n}(\lambda)=(-1)^{n}, n \geq 1$;

(iii) $\lambda \leq \xi$ (in particular, $-\infty<\xi$ and $\lambda$ must be real);

(iv) $(x-\lambda) \sigma$ is positive-definite on $[\xi, \eta]$.

Proof. See Theorem 7.1 in [4, Chapter 1].

Due to (2.3), we call $\left\{P_{n}^{*}(\lambda ; x)\right\}_{n=0}^{\infty}$ the monic kernel polynomial system (MKPS) for $\left\{P_{n}(x)\right\}_{n=0}^{\infty}$ (or for $\sigma$ ) with $K$-parameter $\lambda$. Conversely, we may express $P_{n}(x)$ in terms of $\left\{P_{n}^{*}(\lambda ; x)\right\}_{n=0}^{\infty}$. By (2.3), we have

$$
\begin{aligned}
\frac{P_{n}(x) P_{n}(\lambda)}{<\sigma, P_{n}^{2}>} & =K_{n}(x, \lambda)-K_{n-1}(x, \lambda) \\
& =\frac{P_{n}(\lambda)}{<\sigma, P_{n}^{2}>} P_{n}^{*}(\lambda ; x)-\frac{P_{n-1}(\lambda)}{<\sigma, P_{n-1}^{2}>} P_{n-1}^{*}(\lambda ; x)
\end{aligned}
$$


so that

$$
P_{n}(x)=P_{n}^{*}(\lambda ; x)-\frac{P_{n-1}(\lambda)}{P_{n}(\lambda)} c_{n} P_{n-1}^{*}(\lambda ; x), \quad n \geq 0\left(P_{-1}^{*}(\lambda ; x)=0\right),
$$

where $c_{n}$ are the coefficient of the three-term recurrence relation (2.1) for $\left\{P_{n}(x)\right\}_{n=0}^{\infty}$. Hence $P_{n}(x)$ is quasi-orthogonal (see Definitio 5.1 in [4, Chapter 2]) of order 1 relative to $(x-\lambda) \sigma$. In fact, relation (2.5) characterizes kernel polynomials completely as we shall see later (see Theorem 3.2).

\section{Kernel polynomials}

By Proposition 2.5, we see that for any MOPS $\left\{P_{n}(x)\right\}_{n=0}^{\infty}$ relative to $\sigma$ and any $K$-parameter $\lambda$ with $P_{n}(\lambda) \neq 0, n \geq 1$, its $\operatorname{MKPS}\left\{P_{n}^{*}(\lambda ; x)\right\}_{n=0}^{\infty}$ is uniquely determined by (2.3). We now ask: when is a given MOPS $\left\{Q_{n}(x)\right\}_{n=0}^{\infty}$ also an MKPS for some other MOPSs?

Lemma 3.1. Let $\sigma$ be a quasi-definite moment functional and $u=\sigma+c \delta(x-\lambda)$ $(c, \lambda \in \mathbb{C})$. Then, $u$ is also quasi-definite if and only if

$$
d_{n}:=1+c K_{n}(\lambda, \lambda) \neq 0, \quad n \geq 0,
$$

where $K_{n}(x, y)$ is the $n$-th kernel polynomial for $\sigma$. In this case, the monic OPS $\left\{R_{n}(x)\right\}_{n=0}^{\infty}$ relative to $u$ is given by

$$
R_{n}(x)=P_{n}(x)-c \frac{P_{n}(\lambda)}{d_{n-1}} K_{n-1}(x, \lambda), \quad n \geq 0\left(d_{-1}=1, K_{-1}(x, y)=0\right) .
$$

Proof. See Corollary 3.2 in [10] (see also [11]).

Theorem 3.2. For an MOPS $\left\{Q_{n}(x)\right\}_{n=0}^{\infty}$, the following are all equivalent:

(i) $\left\{Q_{n}(x)\right\}_{n=0}^{\infty}$ is an MKPS for some other OPS;

(ii) there are complex numbers $\lambda, a_{n} \neq 0(n \geq 0)$, and an MOPS $\left\{P_{n}(x)\right\}_{n=0}^{\infty}$ such that

$$
(x-\lambda) Q_{n}(x)=P_{n+1}(x)-a_{n} P_{n}(x), \quad n \geq 0 ;
$$

(iii) there are complex numbers $\alpha_{n}(n \geq 1)$ such that $\alpha_{1} \neq 0$ and

$$
Q_{n}(x)-\alpha_{n} Q_{n-1}(x), \quad n \geq 0 \quad\left(Q_{-1}(x)=0\right),
$$

form an MOPS.

In this case, $\left\{Q_{n}(x)\right\}_{n=0}^{\infty}$ is an MKPS for a quasi-definite moment functional $\tau$ with $K$-parameter $\lambda$ if and only if

$$
\tau=a \sigma+b \delta(x-\lambda),
$$

where $\sigma$ is an orthogonalizing moment functional for $\left\{P_{n}(x)\right\}_{n=0}^{\infty}$ in (ii) and a $(\neq 0)$ and $b$ are complex numbers satisfying

$$
a+b K_{n}(\lambda, \lambda) \neq 0, \quad n \geq 0,
$$

where $K_{n}(x, y)$ is the $n$-th kernel polynomial for $\sigma$. 
Proof. (i) $\Rightarrow$ (ii): Assume $\left\{Q_{n}(x)\right\}_{n=0}^{\infty}=\left\{P_{n}^{*}(\lambda ; x)\right\}_{n=0}^{\infty}$. Then we have (3.1) with $a_{n}=\frac{P_{n+1}(\lambda)}{P_{n}(\lambda)}$ by Proposition 2.5 .

(ii) $\Rightarrow$ (i): Assume that (ii) holds. Then

$$
\begin{aligned}
\left\langle(x-\lambda) \sigma, x^{k} Q_{n}(x)\right\rangle & =\left\langle\sigma, x^{k}\left(P_{n+1}(x)-a_{n} P_{n}(x)\right)\right\rangle \\
& =-a_{n}\left\langle\sigma, P_{n}^{2}\right\rangle \delta_{k n}, \quad 0 \leq k \leq n,
\end{aligned}
$$

so that $\left\{Q_{n}(x)\right\}_{n=0}^{\infty}$ is an MOPS relative to $(x-\lambda) \sigma$. Hence, $\left\{Q_{n}(x)\right\}_{n=0}^{\infty}=$ $\left\{P_{n}^{*}(\lambda ; x)\right\}_{n=0}^{\infty}$ by Proposition 2.5 .

(i) $\Rightarrow$ (iii): This follows immediately from (2.5).

(iii) $\Rightarrow$ (i): See Theorem 4.2.

Now, assume $\left\{Q_{n}(x)\right\}_{n=0}^{\infty}=\left\{P_{n}^{*}(\lambda ; x)\right\}_{n=0}^{\infty}$. If $\left\{Q_{n}(x)\right\}_{n=0}^{\infty}$ is also an MKPS for $\tau$ with $K$-parameter $\lambda$, then $\left\{Q_{n}(x)\right\}_{n=0}^{\infty}$ is an MOPS relative to $(x-\lambda) \sigma$ and $(x-\lambda) \tau$. Hence $(x-\lambda) \tau=a(x-\lambda) \sigma$ for some $a \neq 0$ so that we have (3.2) with $b=\tau_{0}$. Since $\tau$ is quasi-def nite, (3.3) follows from Lemma 3.1. Conversely, if $\tau$ is given by (3.2) and (3.3) holds, then $\tau$ is quasi-def nite by Lemma 3.1 and $(x-\lambda) \tau=a(x-\lambda) \sigma$. Hence $\left\{Q_{n}(x)\right\}_{n=0}^{\infty}$ is an MOPS relative to $(x-\lambda) \tau$ (and $(x-\lambda) \sigma)$ so that $\left\{Q_{n}(x)\right\}_{n=0}^{\infty}$ must be an MKPS for $\tau$ with $K$-parameter $\lambda$.

Theorem 3.2 characterizes MKPSs and shows that an MOPS can be an MKPS for inf nitely many distinct MOPSs with the same $K$-parameter. In fact, by Lemma 3.1, if $\left\{Q_{n}(x)\right\}_{n=0}^{\infty}$ is an MKPS for $\left\{P_{n}(x)\right\}_{n=0}^{\infty}$ with $K$-parameter $\lambda$, then $\left\{Q_{n}(x)\right\}_{n=0}^{\infty}$ is also an MKPS for $\left\{P_{n}(x)-\frac{b}{a} \cdot \frac{P_{n}(\lambda)}{d_{n-1}} K_{n-1}(x, \lambda)\right\}_{n=0}^{\infty}$ with $K$-parameter $\lambda$ for any complex numbers $a \neq 0$ and $b$ satisfying (3.3).

Quasi-orthogonality relations like (2.5) and (3.1) imply that there are close relations for relative locations of zeros of $\left\{P_{n}(x)\right\}_{n=0}^{\infty}$ and $\left\{P_{n}^{*}(\lambda ; x)\right\}_{n=0}^{\infty}$ when they are real polynomials and one of them is positive-def nite.

Theorem 3.3. Let $\left\{P_{n}(x)\right\}_{n=0}^{\infty}$ be an MOPS relative to a positive-definite moment functional $\sigma$ and $[\xi, \eta]$ the true interval of orthogonality of $\sigma$. If $\lambda$ is any real number such that $P_{n}(\lambda) \neq 0, n \geq 1$, then $P_{n}^{*}(\lambda ; x)$ has $n$ real simple zeros, which interlace with zeros of $P_{n}(x)$ and $P_{n+1}(x)$. More precisely, let $x_{n, 1}<x_{n, 2}<\cdots<x_{n, n}$ and $x_{n, 1}^{*}<x_{n, 2}^{*}<\ldots<x_{n, n}^{*}$ be zeros of $P_{n}(x)$ and $P_{n}^{*}(\lambda ; x)$ respectively and $x_{n, 0}=\xi$, $x_{n, n+1}=\eta$.

(i) If $\lambda \leq \xi$, then $P_{n}^{*}(\lambda ; x)$ has one zero in each $\left(x_{n, k}, x_{n+1, k+1}\right), k=1,2, \ldots, n$.

(ii) If $\lambda \in\left(x_{n, k-1}, x_{n+1, k}\right)$ for some $k=1,2, \ldots, n+1$, then $P_{n}^{*}(\lambda ; x)$ has one zero in each one of $\left(-\infty, x_{n+1,1}\right)$ and $\left(x_{n, j-1}, x_{n+1, j}\right), j=2,3, \ldots, k-1$, $k+1, \ldots, n+1$.

(iii) If $\lambda \in\left(x_{n+1, k}, x_{n, k}\right)$ for some $k=1,2, \ldots, n+1$, then $P_{n}^{*}(\lambda ; x)$ has one zero in each one of $\left(x_{n+1, j}, x_{n, j}\right), j=1,2, \ldots, k-1, k+1, \ldots, n$ and $\left(x_{n+1, n+1}, \infty\right)$.

(iv) If $\lambda \geq \eta$, then $P_{n}^{*}(\lambda ; x)$ has one zero in each $\left(x_{n+1, k}, x_{n, k}\right), k=1,2, \ldots, n$.

Proof. We shall prove only (ii); the proofs for (i),(iii), and (iv) are similar. Assume $\lambda \in\left(x_{n, k-1}, x_{n+1, k}\right)$ for some $1 \leq k \leq n+1$. Using

$$
\begin{aligned}
& \operatorname{sgn} P_{n}(-\infty)=(-1)^{n}, \quad \operatorname{sgn} P_{n}\left(x_{n+1, j}\right)=\operatorname{sgn} P_{n+1}\left(x_{n, j}\right)=(-1)^{n+1-j}, \\
& 1 \leq j \leq n+1, \quad \operatorname{sgn} P_{n}(\infty)=+1,
\end{aligned}
$$


we can easily see that $\operatorname{sgn} P_{n}(\lambda) P_{n+1}(\lambda)=-1$ for $\lambda \in\left(x_{n, k-1}, x_{n+1, k}\right)$. By (2.3),

$$
P_{n}^{*}\left(\lambda ; x_{n, j-1}\right)=\left(x_{n, j-1}-\lambda\right)^{-1} P_{n+1}\left(x_{n, j-1}\right), \quad 2 \leq j \leq n+1,
$$

so that

$$
\operatorname{sgn} P_{n}^{*}\left(\lambda ; x_{n, j-1}\right)= \begin{cases}(-1)^{n+1-j}, & 2 \leq j \leq k \\ (-1)^{n-j}, & k+1 \leq j \leq n+1\end{cases}
$$

and

$$
P_{n}^{*}\left(\lambda ; x_{n+1, j}\right)=-\left(x_{n+1, j}-\lambda\right)^{-1} \frac{P_{n+1}(\lambda)}{P_{n}(\lambda)} P_{n}\left(x_{n+1, j}\right), \quad 1 \leq j \leq n+1,
$$

so that

$$
\operatorname{sgn} P_{n}^{*}\left(\lambda ; x_{n+1, j}\right)= \begin{cases}(-1)^{n-j}, & 1 \leq j \leq k-1 \\ (-1)^{n+1-j}, & k \leq j \leq n+1 .\end{cases}
$$

Hence, $P_{n}^{*}\left(\lambda ; x_{n, j-1}\right) P_{n}^{*}\left(\lambda ; x_{n+1, j}\right)<0$ for $j=2,3, \ldots, k-1, k+1, \ldots, n+1$. Finally, $\operatorname{sgn} P_{n}^{*}(\lambda ;-\infty)=(-1)^{n}$ so that $P_{n}^{*}(\lambda ;-\infty) P_{n}^{*}\left(x_{n+1,1}\right)<0$. Hence, the conclusion follows.

In the case $\lambda \leq \xi$ (and $\lambda \geq \eta),(x-\lambda) \sigma$ (respectively $(\lambda-x) \sigma)$ is positivedef nite and Theorem 3.3 (i) was proved in [4]. Note that when $\xi<\lambda<\eta$, $\left\{P_{n}^{*}(\lambda ; x)\right\}_{n=0}^{\infty}$ is never positive-defi ite. However, $\left\{P_{n}^{*}(\lambda ; x)\right\}_{n=0}^{\infty}$ can be positivedefi ite even if $\left\{P_{n}(x)\right\}_{n=0}^{\infty}$ is not positive-def nite (cf. Theorem 4.4).

Theorem 3.4. Let $\left\{P_{n}^{*}(\lambda ; x)\right\}_{n=0}^{\infty}$ be an MKPS for a real MOPS $\left\{P_{n}(x)\right\}_{n=0}^{\infty}$ with real $K$-parameter $\lambda$. If $\left\{P_{n}^{*}(\lambda ; x)\right\}_{n=0}^{\infty}$ is positive-definite, then $P_{n}(x)$ has $n$ real simple zeros, which interlace with zeros of $P_{n-1}^{*}(\lambda ; x)$ and $P_{n}^{*}(\lambda ; x)$. To be more precise, we have

$$
x_{n-1, k-1}^{*}<x_{n, k}<x_{n, k}^{*}, \quad 1 \leq k \leq n \quad\left(x_{n-1,0}^{*}=-\infty\right),
$$

if $c_{n} P_{n}(\lambda) P_{n-1}(\lambda)<0$ and

$$
x_{n, k}^{*}<x_{n, k}<x_{n-1, k}^{*}, \quad 1 \leq k \leq n \quad\left(x_{n-1, n}=\infty\right),
$$

if $c_{n} P_{n-1}(\lambda) P_{n}(\lambda)>0$.

Proof. It's a straightforward consequence of the relation (2.5) and (3.4).

Finally in this section, let's consider the semi-classical character of MKPSs.

Lemma 3.5. Let $\sigma$ be a semi-classical moment functional satisfying the equation (2.2) and

$$
s:=\max (\operatorname{deg}(\alpha)-2, \operatorname{deg}(\beta)-1) .
$$

Then, $\sigma$ is of class $s$ if and only if for any zero $c$ of $\alpha(x)$

$$
\left|r_{c}\right|+\left|\left\langle\sigma, q_{c}(x)\right\rangle\right| \neq 0,
$$

where $\alpha(x)=(x-c) \alpha_{c}(x)$ and $\alpha_{c}(x)-\beta(x)=(x-c) q_{c}(x)+r_{c}$. 
Proof. See Proposition 3.5 in [13].

Theorem 3.6. If $\left\{P_{n}(x)\right\}_{n=0}^{\infty}$ is a SCOPS of class s relative to $\sigma$ satisfying (2.2) and (3.5), then $\left\{P_{n}^{*}(\lambda ; x)\right\}_{n=0}^{\infty}$ is a SCOPS of class $s-1$ when $\alpha(\lambda)=\beta(\lambda)=0$, s when $\alpha(\lambda)=0$ and $\beta(\lambda) \neq 0$, and $s+1$ when $\alpha(\lambda) \neq 0$. Conversely, if $\left\{P_{n}^{*}(\lambda ; x)\right\}_{n=0}^{\infty}$ is a SCOPS of class $s$, then $\left\{P_{n}(x)\right\}_{n=0}^{\infty}$ is a SCOPS of class $s-1$ or $s$ or $s+1$.

Proof. Let $\tau=(x-\lambda) \sigma$ and $\alpha(x)=(x-c) \alpha_{c}(x), \alpha_{c}(x)-\beta(x)=(x-c) q_{c}(x)+r_{c}$ for any zero $c$ of $\alpha(x)$.

(a) Assume $\alpha(\lambda)=\beta(\lambda)=0$. Then $\left(\alpha_{\lambda}(x) \tau\right)^{\prime}=\beta_{\lambda}(x) \tau$, where $\beta(x)=(x-$ $\lambda) \beta_{\lambda}(x)$ so that $\tau$ is of class $\leq s-1$. Let $\alpha_{\lambda}(x)=(x-c) \alpha_{\lambda, c}(x)$ and $\alpha_{\lambda, c}(x)-$ $\beta_{\lambda}(x)=(x-c) q_{\lambda, c}(x)+r_{\lambda, c}$, for any zero $c$ of $\alpha_{\lambda}(x)$. If $\alpha_{\lambda}(\lambda)=0$, then $\left(\alpha_{\lambda} \sigma\right)^{\prime}=\left(\beta_{\lambda}-\alpha_{\lambda, \lambda}\right) \sigma$ so that $\sigma$ is of class $\leq s-1$, which is a contradiction. Hence $\alpha_{\lambda}(\lambda) \neq 0$, that is, $c \neq \lambda$. Now, we have

$$
r_{c}=\alpha_{c}(c)-\beta(c)=(c-\lambda) r_{\lambda, c} .
$$

Hence, if $r_{\lambda, c}=0$, then $r_{c}=0,\left\langle\sigma, q_{c}\right\rangle \neq 0$, and

$$
q_{\lambda, c}(x)=\frac{\alpha_{\lambda, c}(x)-\beta_{\lambda}(x)}{x-c}=\frac{1}{x-\lambda}\left[\frac{\alpha(x)}{(x-c)^{2}}-\frac{\beta(x)}{x-c}\right]=\frac{q_{c}(x)}{x-\lambda},
$$

so that $\left\langle\tau, q_{\lambda, c}\right\rangle=\left\langle\sigma,(x-\lambda) q_{\lambda, c}\right\rangle=\left\langle\sigma, q_{c}\right\rangle \neq 0$. Therefore, $\tau$ is of class $s-1$ by Lemma 3.5 .

(b) Assume $\alpha(\lambda)=0$ and $\beta(\lambda) \neq 0$. Then $(\alpha \tau)^{\prime}=\left(\alpha_{\lambda}+\beta\right) \tau$ so that $\tau$ is of class $\leq s$. Let $\alpha_{c}(x)-\alpha_{\lambda}(x)-\beta(x)=(x-c) \tilde{q}_{c}(x)+\tilde{r}_{c}$ for any zero $c$ of $\alpha(x)$. First assume $c \neq \lambda$. Then $\alpha_{\lambda}(c)=0$ so that $\tilde{r}_{c}=\alpha_{c}(c)-\beta(c)=r_{c}$. Hence if $\tilde{r}_{c}=0$, then $r_{c}=0,\left\langle\sigma, q_{c}\right\rangle \neq 0$, and

$$
\tilde{q}_{c}(x)=q_{c}(x)-\frac{\alpha_{\lambda}(x)}{x-c} .
$$

Hence

$$
\begin{gathered}
\left\langle\tau, \tilde{q}_{c}\right\rangle=\left\langle\sigma,(x-\lambda)\left(q_{c}(x)-\frac{\alpha_{\lambda}(x)}{x-c}\right)\right\rangle=\left\langle\sigma,(x-\lambda) q_{c}(x)-\alpha_{c}(x)\right\rangle \\
=\left\langle\sigma,(x-c+c-\lambda) q_{c}(x)-\alpha_{c}(x)\right\rangle=(c-\lambda)\left\langle\sigma, q_{c}\right\rangle+\langle\sigma, \beta\rangle \\
=(c-\lambda)\left\langle\sigma, q_{c}\right\rangle \neq 0
\end{gathered}
$$

since $\langle\sigma, \beta\rangle=\langle\beta \sigma, 1\rangle=\left\langle(\alpha \sigma)^{\prime}, 1\right\rangle=0$ and $(x-c) q_{c}(x)=\alpha_{c}(x)-\beta(x)$. Now if $c=\lambda$, then $\tilde{r}_{c}=-\beta(\lambda) \neq 0$. Therefore, $\tau$ is of class $s$ by Lemma 3.5.

(c) Assume $\alpha(\lambda) \neq 0$. Then $(\tilde{\alpha} \tau)^{\prime}=\tilde{\beta} \tau$, where $\tilde{\alpha}(x)=(x-c) \alpha(x)$ and $\tilde{\beta}(x)=$ $2 \alpha(x)+(x-\lambda) \beta(x)$, so that $\tau$ is of class $\leq s+1$. As before, let $\tilde{\alpha}(x)=$ $(x-c) \tilde{\alpha}_{c}(x)$ and $\tilde{\alpha}_{c}(x)-\tilde{\beta}(x)=(x-c) \tilde{q}_{c}(x)+\tilde{r}_{c}$ for any zero $c$ of $\alpha(x)$. If $c=\lambda$, then $\tilde{\alpha}_{\lambda}(x)=\alpha(x)$ so that $\tilde{r}_{\lambda}=-\alpha(\lambda) \neq 0$. Now assume $c \neq \lambda$. Then $\tilde{\alpha}_{c}(x)=(x-\lambda) \alpha_{c}(x)$ so that $\tilde{r}_{c}=\tilde{\alpha}_{c}(c)-\tilde{\beta}(c)=(c-\lambda) r_{c}$. Hence, if $\tilde{r}_{c}=0$, then $r_{c}=0,\left\langle\sigma, q_{c}\right\rangle \neq 0$, and $\tilde{q}_{c}(x)=\frac{\tilde{\alpha}_{c}(x)-\tilde{\beta}(x)}{x-c}=(x-\lambda) q_{c}(x)-2 \alpha_{c}(x)=(c-\lambda) q_{c}(x)-\beta(x)-\alpha_{c}(x)$, 
so that

$$
\begin{gathered}
\left\langle\tau, \tilde{q}_{c}\right\rangle=\left\langle\sigma,(x-\lambda) \tilde{q}_{c}\right\rangle=\left\langle\sigma,(x-c+c-\lambda)\left\{(c-\lambda) q_{c}(x)-\beta(x)-\alpha_{c}(x)\right\}\right\rangle \\
=\left\langle\sigma,(c-\lambda)^{2} q_{c}\right\rangle-\langle\sigma, \alpha\rangle-\langle\sigma,(x+c-2 \lambda) \beta\rangle=(c-\lambda)^{2}\left\langle\sigma, q_{c}\right\rangle \neq 0
\end{gathered}
$$

since $(x-c) q_{c}(x)=\alpha_{c}(x)-\beta(x),\langle\sigma, \beta\rangle=0$, and $\langle\sigma, x \beta\rangle=-\langle\sigma, \alpha\rangle$. Therefore, $\tau$ is of class $s+1$ by Lemma 3.5.

Now, the converse is trivial.

The last case when $\alpha(\lambda) \neq 0$ was proved by Belmehdi (see Theorem 3.1 in [1]), where the structure relation for $\left\{P_{n}^{*}(\lambda ;, x)\right\}_{n=0}^{\infty}$ is also given.

As a consequence of Theorem 3.6, we have: if $\left\{P_{n}(x)\right\}_{n=0}^{\infty}$ is a classical OPS satisfying a second-order linear differential equation

$$
\alpha(x) P_{n}^{\prime \prime}(x)+\beta(x) P_{n}^{\prime}(x)=\lambda_{n} P_{n}(x), \quad n \geq 0,
$$

where $\alpha(x)$ and $\beta(x)$ are polynomials with $\operatorname{deg}(\alpha) \leq 2$ and $\operatorname{deg}(\beta)=1$, then $\alpha^{2}(x)+\beta^{2}(x) \neq 0$ and for any $\lambda$ with $P_{n}(\lambda) \neq 0, n \geq 1,\left\{P_{n}^{*}(\lambda ; x)\right\}_{n=0}^{\infty}$ is a SCOPS of class 0 if $\alpha(\lambda)=0$ or of class 1 if $\alpha(\lambda) \neq 0$. Moreover, $\left\{P_{n}^{*}(\lambda ; x)\right\}_{n=0}^{\infty}$ is also a classical OPS only when $\left\{P_{n}(x)\right\}_{n=0}^{\infty}$ is the Jacobi polynomial sequence with $\lambda= \pm 1$ or Bessel or Laguerre polynomial sequences with $\lambda=0$. For example, the MKPS for Laguerre polynomials $\left\{L_{n}^{(\alpha)}(x)\right\}_{n=0}^{\infty}$ with $K$-parameter 0 is $\left\{L_{n}^{(\alpha+1)}(x)\right\}_{n=0}^{\infty}$. Note also that $\left\{P_{n}^{*}(\lambda ; x)\right\}_{n=0}^{\infty}$ may be a classical OPS even if $\left\{P_{n}(x)\right\}_{n=0}^{\infty}$ is not a classical OPS but a SCOPS of class 1. For example, let $\left\{P_{n}(x)\right\}_{n=0}^{\infty}$ be one of the three classical-type OPSs, which are eigenfunctions of the fourth-order linear differential equations

$$
\sum_{i=1}^{4} \ell_{i}(x) y^{(i)}(x)=\lambda_{n} y(x),
$$

where $\ell_{i}(x)$ is a polynomial of degree $\leq i$ and $\ell_{4}(x) \neq 0$. Then we know (see $\left.[7,8]\right)$ that $\left\{P_{n}(x)\right\}_{n=0}^{\infty}$ is orthogonal with respect to $\tau=\sigma+a \delta(x-\lambda)$, where $\sigma$ is a classical moment functional satisfying $(\alpha(x) \sigma)^{\prime}=\beta(x) \sigma$ and $\alpha(\lambda)=0$. Hence $\tau$ satisfie

$$
[(x-\lambda) \alpha(x) \tau]^{\prime}=[(x-\lambda) \beta(x)-\alpha(x)] \tau
$$

so that $\tau$ is a semi-classical moment functional of class 1 (cf. Theorem 5.2 in [10]). Therefore, by Theorem 3.6, $\left\{P_{n}^{*}(\lambda ; x)\right\}_{n=0}^{\infty}$ must be a classical OPS relative to $(x-\lambda) \tau=(x-\lambda) \sigma$. For example, the differential equation

$$
\begin{aligned}
x^{2} y^{(4)}+\left(4 x-2 x^{2}\right) y^{(3)} & +\left[x^{2}-(2 R+6) x\right] y^{\prime \prime}+[(2 R+2) x-2 R] y^{\prime} \\
& =[(2 R+2) n+n(n-1)] y
\end{aligned}
$$

has an OPS $\left\{L_{n}(R ; x)\right\}_{n=0}^{\infty}$, called the Laguerre-type OPS, as solutions for $R \neq$ $0,-1,-2, \ldots$ Since $\left\{L_{n}(R ; x)\right\}_{n=0}^{\infty}$ is an OPS relative to $\left(e^{-x}+\frac{1}{R} \delta(x)\right) d x$ on $[0, \infty)$, its MKPS with $K$ - parameter 0 are the Laguerre polynomials $\left\{L_{n}^{(1)}(x)\right\}_{n=0}^{\infty}$. 


\section{Self-perturbation of orthogonal polynomials}

Relation (2.5) for an MOPS and its MKPS leads to a question: given an MOPS $\left\{Q_{n}(x)\right\}_{n=0}^{\infty}$ and a sequence of complex numbers $\left\{\alpha_{n}\right\}_{n=0}^{\infty}$, def ne another monic PS by

$$
P_{n}(x)=Q_{n}(x)-\alpha_{n} Q_{n-t}(x), \quad n \geq 0,
$$

where $t$ is a positive integer and $Q_{-t}(x)=Q_{1-t}(x)=\ldots=Q_{-1}(x)=0$ (so that the choices of $\alpha_{0}, \alpha_{1}, \ldots, \alpha_{t-1}$ are redundant). When is $\left\{P_{n}(x)\right\}_{n=0}^{\infty}$ also an MOPS? In the following, we always let

$$
\begin{gathered}
P_{n+1}(x)=\left(x-b_{n}\right) P_{n}(x)-c_{n} P_{n-1}(x), \quad n \geq 0 \quad\left(c_{n} \neq 0, n \geq 1\right) \\
Q_{n+1}(x)=\left(x-\tilde{b}_{n}\right) Q_{n}(x)-\tilde{c}_{n} Q_{n-1}(x), \quad n \geq 0 \quad\left(\tilde{c}_{n} \neq 0, n \geq 1\right)
\end{gathered}
$$

be the three-term recurrence relations for $\left\{P_{n}(x)\right\}_{n=0}^{\infty}$ and $\left\{Q_{n}(x)\right\}_{n=0}^{\infty}$, respectively, when they are MOPSs.

By (4.1) and (4.3), (4.2) gives

$$
\begin{aligned}
& \left(\tilde{b}_{n}-b_{n}\right) Q_{n}(x)+\left(\tilde{c}_{n}-c_{n}\right) Q_{n-1}(x)+\left(\alpha_{n+1}-\alpha_{n}\right) Q_{n-t+1}(x) \\
& +\left(\alpha_{n} b_{n}-\alpha_{n} \tilde{b}_{n-t}\right) Q_{n-t}(x)+\left(\alpha_{n-1} c_{n}-\alpha_{n} \tilde{c}_{n-t}\right) Q_{n-t-1}(x)=0
\end{aligned}
$$

for $n \geq t+1$. On the other hand, if $\left\{P_{n}(x)\right\}_{n=0}^{\infty}$ is an MOPS relative to $\sigma$ and $\left\{Q_{n}(x)\right\}_{n=0}^{\infty}$ is an MOPS relative to $\tau$, then $\left\langle\tau, P_{n}\right\rangle=\left\langle\tau, Q_{n}-\alpha_{n} Q_{n-t}\right\rangle=0$, $n \geq t+1$ so that by Lemma 2.3

$$
\tau=\phi_{t}(x) \sigma
$$

where $\phi_{t}(x)$ is a polynomial of degree $\leq t$. If we set

$$
\phi_{t}(x)=\sum_{j=0}^{t} a_{j} P_{j}(x),
$$

then

$$
a_{j}=\frac{\left\langle\tau, P_{j}\right\rangle}{\left\langle\sigma, P_{j}^{2}\right\rangle}= \begin{cases}\frac{\tau_{0}}{\sigma_{0}} \neq 0, & j=0 \\ 0, & 1 \leq j \leq t-1 \\ -\frac{\alpha_{t} \tau_{0}}{\left\langle\sigma, P_{t}^{2}\right\rangle}, & j=t\end{cases}
$$

Hence

$$
\phi_{t}(x)=-\frac{\alpha_{t} \tau_{0}}{\left\langle\sigma, P_{t}^{2}\right\rangle} P_{t}(x)+\frac{\tau_{0}}{\sigma_{0}} \quad\left(\sigma_{0}=<\sigma, 1>, \tau_{0}=<\tau, 1>\right),
$$

so that $\operatorname{deg}\left(\phi_{t}\right)=t$ if and only if $\alpha_{t} \neq 0$.

Assume that $\alpha_{t}=0$ and $\left\{P_{n}(x)\right\}_{n=0}^{\infty}$ is an MOPS. Then by (4.5) and (4.6), $\tau=c \sigma$ for some non-zero constant $c$. Hence, $\left\{P_{n}(x)\right\}_{n=0}^{\infty}$ must be an MOPS relative to $\sigma$ and $\tau$ so that $\left\{P_{n}(x)\right\}_{n=0}^{\infty}=\left\{Q_{n}(x)\right\}_{n=0}^{\infty}$, that is, $\alpha_{n}=0, n \geq t$. Hence, from now on, we always assume $\alpha_{t} \neq 0$ so that $\operatorname{deg} \phi_{t}=t$.

Now, by the relation (4.4), we must consider the three cases $t=1, t=2$, and $t \geq 3$ separately. 
Proposition 4.1. If $t \geq 3$, then $\left\{P_{n}(x)\right\}_{n=0}^{\infty}$ defined by (4.1) cannot be an MOPS.

Proof. Assume $t \geq 3$ and $\left\{P_{n}(x)\right\}_{n=0}^{\infty}$ is an MOPS. Then (4.2) for $n=t-1$ becomes, via (4.1) and (4.3),

$$
Q_{t}(x)-\alpha_{t}=Q_{t}(x)+\left(\tilde{b}_{t-1}-b_{t-1}\right) Q_{t-1}(x)+\left(\tilde{c}_{t-1}-c_{t-1}\right) Q_{t-2}(x),
$$

so that $\alpha_{t}=0$, which is a contradiction.

Hence, we only need to consider the cases $t=1$ and $t=2$.

Theorem 4.2. Let $\left\{Q_{n}(x)\right\}_{n=0}^{\infty}$ be an MOPS relative to $\tau$ and define another monic $P S\left\{P_{n}(x)\right\}_{n=0}^{\infty}$ by

$$
P_{n}(x)=Q_{n}(x)-\alpha_{n} Q_{n-1}(x), \quad n \geq 0 \quad\left(P_{-1}(x) \equiv 0\right),
$$

where $\alpha_{n}$ are complex numbers with $\alpha_{1} \neq 0$. Then, the following are all equivalent:

(i) $\left\{P_{n}(x)\right\}_{n=0}^{\infty}$ is an MOPS (respectively, a positive-definite MOPS);

(ii) $\alpha_{n} \neq 0, n \geq 1$ (respectively, $\alpha_{n} \alpha_{n+1} \tilde{c}_{n}>0, n \geq 1$ ) and

$$
\begin{aligned}
& \frac{\tilde{c}_{n}}{\alpha_{n}}+\alpha_{n+1}+\tilde{b}_{n}=\lambda(\text { constant }), \quad n \geq 1, \\
c:= & \left.\lambda-\alpha_{1}-\tilde{b}_{0} \neq 0 \text { (respectively, } c \alpha_{1}>0\right) ;
\end{aligned}
$$

(iii) there are complex numbers $\lambda$ and $c \neq 0$ such that

$$
\begin{aligned}
& Q_{n}(c ; \lambda) \neq 0, \quad n \geq 0 \\
& \left(\text { respectively, } c Q_{1}(c ; \lambda)>0 \text { and } Q_{n-1}(c ; \lambda) Q_{n+1}(c ; \lambda) \tilde{c}_{n}>0, n \geq 1\right)
\end{aligned}
$$

and

$$
\alpha_{n}=\frac{Q_{n}(\lambda)-c Q_{n-1}^{(1)}(\lambda)}{Q_{n-1}(\lambda)-c Q_{n-2}^{(1)}(\lambda)}=\frac{Q_{n}(c ; \lambda)}{Q_{n-1}(c ; \lambda)}, \quad n \geq 1 \quad\left(Q_{-1}^{(1)}(x)=0\right) .
$$

In this case, $\left\{P_{n}(x)\right\}_{n=0}^{\infty}$ is the MOPS relative to

$$
\sigma=(x-\lambda)^{-1} \tau-\frac{\tau_{0}}{c} \delta(x-\lambda),
$$

which is quasi-definite (respectively, positive-definite or negative-definite) and $\left\{Q_{n}(x)\right\}_{n=0}^{\infty}=\left\{P_{n}^{*}(\lambda ; x)\right\}_{n=0}^{\infty}$ is the MKPS for $\left\{P_{n}(x)\right\}_{n=0}^{\infty}$ with K-parameter $\lambda$. Here, we call a moment functional $\sigma$ negative-definite if $-\sigma$ is positive-definite.

Theorem 4.2 was proved by Marcellán and Petronilho when $\left\{P_{n}(x)\right\}_{n=0}^{\infty}$ is a quasi-def nite OPS (cf. Theorem 2 in [12]). But, the equivalent conditions for 
positive-def niteness of $\left\{P_{n}(x)\right\}_{n=0}^{\infty}$ follow immediately from (4.8) and the following relations between coefficient of three-term recurrence relations for $\left\{P_{n}(x)\right\}_{n=0}^{\infty}$ and $\left\{Q_{n}(x)\right\}_{n=0}^{\infty}$ :

$$
\begin{aligned}
b_{n} & =\tilde{b}_{n}+\alpha_{n+1}-\alpha_{n}, \quad n \geq 0 ; \\
c_{n} & =\tilde{c}_{n}+\alpha_{n}\left(\alpha_{n+1}+\tilde{b}_{n}-\alpha_{n}-\tilde{b}_{n-1}\right), \quad n \geq 1 ; \\
\alpha_{n-1} & c_{n}=\alpha_{n} \tilde{c}_{n-1}, \quad n \geq 0 .
\end{aligned}
$$

Note that Theorem 4.2 gives, in particular, a proof of (iii) $\Rightarrow$ (i) in Theorem 3.2.

Maroni [14] considered a problem that is closely related to Theorem 4.2: given a quasi-def nite moment functional $\tau$ and two complex numbers $a$ and $\lambda$, when is the moment functional $\sigma$ given by

$$
\sigma=(x-\lambda)^{-1} \tau+a \delta(x-\lambda)
$$

also quasi-def nite? Equivalently, when does the division problem $(x-\lambda) \sigma=\tau$ have a quasi-def nite moment functional solution $\sigma$ ? It's easy to see (cf. Theorem 4.2 (iii) and $[14$, Theorem 1.1]) that $\sigma$ is quasi-defi ite if and only if

$$
a Q_{n}(\lambda)+\tau_{0} Q_{n-1}^{(1)}(\lambda) \neq 0, \quad n \geq 0 .
$$

Maroni stated condition (4.12) as

$$
a \neq-\frac{\tau_{0} Q_{n-1}^{(1)}(\lambda)}{Q_{n}(\lambda)}, \quad n \geq 0,
$$

which is not true in general since $Q_{n}(\lambda)$ may be 0 for some $n \geq 1$ unless $\sigma$ is positive-def nite. First note that when $\sigma$ is also quasi-definit and $\left\{P_{n}(x)\right\}_{n=0}^{\infty}$ and $\left\{Q_{n}(x)\right\}_{n=0}^{\infty}$ are MOPSs relative to $\sigma$ and $\tau$ respectively, $\left\{Q_{n}(x)\right\}_{n=0}^{\infty}=$ $\left\{P_{n}^{*}(\lambda ; x)\right\}_{n=0}^{\infty}$. Now, construct an MOPS $\left\{P_{n}(x)\right\}_{n=0}^{\infty}$ as $P_{0}(x)=1, P_{1}(x)=x$, and

$$
P_{n+1}(x)=\left(x-b_{n}\right) P_{n}(x)-c_{n} P_{n-1}(x), \quad n \geq 1,
$$

where $c_{1}=-1$ and $c_{n}, n \geq 2$, are arbitrary non-zero constants. We may choose $b_{n}$, $n \geq 1$, so that $P_{n}(1) \neq 0, n \geq 1$. Assume that $\left\{P_{n}(x)\right\}_{n=0}^{\infty}$ is an MOPS relative to $\sigma$ and $\sigma_{0}=1$. Then $\tau=(x-1) \sigma$ is also quasi-definit and the MOPS $\left\{Q_{n}(x)\right\}_{n=0}^{\infty}$ relative to $\tau$ is just $\left\{P_{n}^{*}(1 ; x)\right\}_{n=0}^{\infty}$. Then $Q_{1}(1)=\left\langle\sigma, P_{1}^{2}\right\rangle K_{1}(1,1)=0$ (cf. (2.5)) since $\left\langle\sigma, P_{1}^{2}\right\rangle=c_{1}=-1$.

Proposition 2.5 gives conditions for an MKPS $\left\{P_{n}^{*}(\lambda ; x)\right\}_{n=0}^{\infty}$ to be positivedefi ite when $\left\{P_{n}(x)\right\}_{n=0}^{\infty}$ is positive-defi ite. Conversely, we now have:

Corollary 4.3. In Theorem 4.2, assume that $\tau$ is positive-definite, $\alpha_{n}$ 's are real, and $\left\{P_{n}(x)\right\}_{n=0}^{\infty}$ is an MOPS. Then, the following are all equivalent:

(i) $\left\{P_{n}(x)\right\}_{n=0}^{\infty}$ is a positive-definite OPS;

(ii) either $c>0$ and $\alpha_{n}<0, n \geq 1$ or $c<0$ and $\alpha_{n}>0, n \geq 0$; 
(iii) either $c<0$ and $\operatorname{sgn} Q_{n}(c ; \lambda)=(-1)^{n}, n \geq 0$ or $c>0$ and $Q_{n}(c ; \lambda)>0$, $n \geq 0$;

(iv) either $\sigma$ or $-\sigma$ is positive-definite.

In this case, $\tau$ is positive-definite on the true interval of orthogonality $[\xi, \eta]$ of $\left\{P_{n}(x)\right\}_{n=0}^{\infty}$ so that either $\lambda \leq \xi$ (if $c<0$ ) or $\lambda \geq \eta$ (if $c>0$ ). Hence, in particular, either $-\infty<\xi$ or $\eta<\infty$.

Proof. The equivalences of (i)-(iv) follow immediately from Theorem 4.2. The last conclusion follows from Proposition 2.5 since $\tau=(x-\lambda) \sigma$ is positive-defi ite and either $\sigma$ or $-\sigma$ is positive-defi ite.

Let $\left\{P_{n}(x)\right\}_{n=0}^{\infty}$ be a monic PS def ned by (4.7) and set $\tilde{P}_{n}(x)=\frac{1}{n+1} P_{n+1}^{\prime}(x)$. Then, (4.7) yields

$$
\tilde{P}_{n}(x)=\frac{1}{n+1} Q_{n+1}^{\prime}(x)-\frac{1}{n+1} \alpha_{n} Q_{n}^{\prime}(x), \quad n \geq 0 .
$$

Hence, if $\left\{\tilde{P}_{n}(x)\right\}_{n=0}^{\infty}$ is also an MOPS, then $\left\{\tilde{P}_{n}(x)\right\}_{n=0}^{\infty}$ and $\left\{Q_{n}(x)\right\}_{n=0}^{\infty}$ are the so-called coherent pairs (cf. $[6,12])$.

With respect to this, Marcellán and Petronilho [12] raised a question: given an MOPS $\left\{P_{n}(x)\right\}_{n=0}^{\infty}$, def ne another PS $\left\{Q_{n}(x)\right\}_{n=0}^{\infty}$ recursively by

$$
P_{n}(x)=Q_{n}(x)-\alpha_{n} Q_{n-1}(x)-\beta_{n}, \quad n \geq 0,
$$

where $\alpha_{n}$ and $\beta_{n}$ are complex numbers with $\alpha_{0}=\beta_{0}=\beta_{1}=0$. When is $\left\{Q_{n}(x)\right\}_{n=0}^{\infty}$ also an MOPS? In the following, we also assume $\alpha_{1} \neq 0$ as before:

Theorem 4.4. Let $\left\{P_{n}(x)\right\}_{n=0}^{\infty}$ be an MOPS satisfying (4.2) and define $\left\{Q_{n}(x)\right\}_{n=0}^{\infty}$ by (4.13). Then, the following are all equivalent:

(i) $\left\{Q_{n}(x)\right\}_{n=0}^{\infty}$ is an MOPS (respectively, a positive-definite MOPS);

(ii) $\beta_{n}=0, n \geq 0$ and there is a complex number $\lambda$ such that $P_{n}(\lambda) \neq 0, n \geq 1$ (respectively, $P_{n-1}(\lambda) P_{n+1}(\lambda) c_{n} c_{n+1}>0, n \geq 1$ ) such that

$$
\alpha_{n}=\frac{P_{n-1}(\lambda)}{P_{n}(\lambda)} c_{n}, \quad n \geq 1 ;
$$

(iii) $\beta_{n}=0, n \geq 0, \alpha_{n} \neq 0, n \geq 1$, (respectively, $\alpha_{n} \alpha_{n+1} c_{n+1}>0, n \geq 1$ ), and

$$
\frac{c_{n}}{\alpha_{n}}+\alpha_{n-1}+b_{n-1}=\lambda \text { (constant), } \quad n \geq 1\left(a_{0}=0\right) \text {. }
$$

In this case, $\left\{Q_{n}(x)\right\}_{n=0}^{\infty}$ is the MKPS for $\left\{P_{n}(x)\right\}_{n=0}^{\infty}$ with $K$-parameter $\lambda$, which is orthogonal with respect to $(x-\lambda) \sigma$ and satisfy the three-term recurrence relation (4.3) with $\tilde{b}_{n}$ and $\tilde{c}_{n}$ determined by (4.9) and (4.10).

Proof. See Theorem 1 in [12].

Theorem 4.4, in particular, shows that $\left\{P_{n}^{*}(\lambda ; x)\right\}_{n=0}^{\infty}$ may be positive-defi ite even if $\left\{P_{n}(x)\right\}_{n=0}^{\infty}$ is not positive-def nite. For example, if $c_{1}<0, c_{n+1}>0, n \geq 1$ and $\alpha_{n} \alpha_{n+1}>0, \quad n \geq 0$, then $\left\{P_{n}(x)\right\}_{n=0}^{\infty}$ is not positive-defi ite but $\left\{Q_{n}(x)\right\}_{n=0}^{\infty}$ is positive-defi ite. As in Corollary 4.3, we also have: 
Corollary 4.5. In Theorem 4.4, assume that $\sigma$ is positive-definite and $\left\{Q_{n}(x)\right\}_{n=0}^{\infty}$ is an MOPS. Then, the following are all equivalent:

(i) $\left\{Q_{n}(x)\right\}_{n=0}^{\infty}$ is a positive-definite OPS;

(ii) either $\alpha_{n}>0, n \geq 1$ or $\alpha_{n}<0, n \geq 1$;

(iii) either $\tau=(x-\lambda) \sigma$ or $-\tau$ is positive-definite on $[\xi, \eta]$;

(iv) either $\lambda \leq \xi$ or $\lambda \geq \xi$,

where $[\xi, \eta]$ is the true interval of orthogonality for $\sigma$.

Remark 4.6. Let $\left\{P_{n}(x)\right\}_{n=0}^{\infty}$ and $\left\{Q_{n}(x)\right\}_{n=0}^{\infty}$ be two real PSs satisfying the relation (4.7). If $\left\{Q_{n}(x)\right\}_{n=0}^{\infty}$ is a positive-definit OPS, then each $P_{n}(x), n \geq 1$, has $n$ real simple zeros, which interlace with the zeros of $Q_{n}(x)$ and with the zeros of $Q_{n-1}(x)$. Here, $\left\{P_{n}(x)\right\}_{n=0}^{\infty}$ need not be an MOPS (cf. Theorem 3.4).

If $\left\{P_{n}(x)\right\}_{n=0}^{\infty}$ is a positive-defi ite OPS and $\left\{Q_{n}(x)\right\}_{n=0}^{\infty}$ is an MOPS, then each $Q_{n}(x), n \geq 1$, has $n$ real simple zeros (cf. Theorem 3.3).

If $\left\{P_{n}(x)\right\}_{n=0}^{\infty}$ is a positive-defi ite OPS but $\left\{Q_{n}(x)\right\}_{n=0}^{\infty}$ is not an OPS, then $Q_{n}(x), n \geq 1$, need not have real or simple zeros. For example, by Wendroff's theorem [15], there is a positive-defi ite OPS $\left\{P_{n}(x)\right\}_{n=0}^{\infty}$ with $P_{0}(x)=1, P_{1}(x)=$ $x$ and $P_{2}(x)=x^{2}-1$. Def ne a PS $\left\{Q_{n}(x)\right\}_{n=0}^{\infty}$ by (4.7), where $\alpha_{n}, n \geq 1$, are real constants. Then $Q_{2}(x)=x^{2}+\alpha_{2} x+\alpha_{1} \alpha_{2}-1$ so that $Q_{2}(x)$ may have two distinct real zeros or one double zero or two complex conjugate zeros.

We now consider the case $t=2$.

Theorem 4.7. Let $\left\{Q_{n}(x)\right\}_{n=0}^{\infty}$ be an MOPS relative to $\tau$ and define another monic $P S\left\{P_{n}(x)\right\}_{n=0}^{\infty}$ by

$$
P_{n}(x)=Q_{n}(x)-\alpha_{n} Q_{n-2}(x), \quad n \geq 0,
$$

where $\alpha_{n}$ are complex numbers with $\alpha_{2} \neq 0$. Then, the following are all equivalent:

(i) $\left\{P_{n}(x)\right\}_{n=0}^{\infty}$ is an MOPS (respectively, a positive-definite MOPS);

(ii) $\alpha_{n} \neq 0, n \geq 2$ and

$$
\begin{aligned}
\tilde{c}_{1}+\alpha_{2} & \neq 0 \quad\left(\text { respectively, } \tilde{c}_{1}+\alpha_{2}>0\right), \\
\tilde{c}_{2}+\alpha_{3}-\alpha_{2} & \neq 0 \quad\left(\text { respectively, } \tilde{c}_{2}+\alpha_{3}-\alpha_{2}>0\right), \\
\tilde{c}_{n}+\alpha_{n+1}-\alpha_{n} & =\frac{\alpha_{n}}{\alpha_{n-1}} \tilde{c}_{n-2}, \quad n \geq 3
\end{aligned}
$$

$$
\begin{aligned}
& \text { (respectively, } \left.\tilde{c}_{n}+\alpha_{n+1}-\alpha_{n}=\frac{\alpha_{n}}{\alpha_{n-1}} \tilde{c}_{n-2}>0, \quad n \geq 3\right), \\
& \tilde{b}_{n}=\tilde{b}_{n-2}, \quad n \geq 2 .
\end{aligned}
$$

In this case, $\left\{P_{n}(x)\right\}_{n=0}^{\infty}$ satisfy the three-term recurrence relation (4.2), where

$$
b_{n}=\tilde{b}_{n}, \quad n \geq 0 \quad \text { and } \quad c_{1}=\tilde{c}_{1}+\alpha_{2}, \quad c_{n}=\tilde{c}_{n}, n \geq 2,
$$

and is orthogonal with respect to $\sigma$ satisfying

$$
\left[x^{2}-\left(\tilde{b}_{0}+\tilde{b}_{1}\right) x+\tilde{b}_{1} \tilde{b}_{2}-\mu\right] \sigma=\tau,
$$

where $\mu=\alpha_{n}\left(1+\frac{\tilde{c}_{n-1}}{\alpha_{n}}\right)\left(1+\frac{\tilde{c}_{n-2}}{\alpha_{n-1}}\right) \neq 0, n \geq 3$. 
Proof. Assume $\left\{P_{n}(x)\right\}_{n=0}^{\infty}$ is an MOPS satisfying the three-term recurrence relation (4.2). For $n=2$ and $n=1$, we have, from (4.2) via (4.14) and (4.3):

$$
\begin{aligned}
& \left(\tilde{b}_{2}-b_{2}\right) Q_{2}(x)+\left(\tilde{c}_{2}-c_{2}+\alpha_{3}-\alpha_{2}\right) Q_{1}(x)+\left(\alpha_{2} b_{2}-\alpha_{2} \tilde{b}_{0}+b_{0} c_{2}-\tilde{b}_{0} c_{2}\right)=0 ; \\
& \left(\tilde{b}_{1}-b_{1}+\tilde{b}_{0}-b_{0}\right) Q_{1}(x)+\tilde{c}_{1}-c_{1}+\alpha_{2}+\tilde{b}_{0}^{2}-b_{0} \tilde{b}_{0}-\tilde{b}_{0} b_{1}+b_{0} b_{1}=0 .
\end{aligned}
$$

On the other hand, for $t=2$ (4.4) becomes

$$
\begin{aligned}
& \left(\tilde{b}_{n}-b_{n}\right) Q_{n}(x)+\left(\tilde{c}_{n}-c_{n}+\alpha_{n+1}-\alpha_{n}\right) Q_{n-1}(x) \\
& +\left(\alpha_{n} b_{n}-\alpha_{n} \tilde{b}_{n-2}\right) Q_{n-2}(x)+\left(\alpha_{n-1} c_{n}-\alpha_{n} \tilde{c}_{n-2}\right) Q_{n-3}(x)=0, \quad n \geq 3 .
\end{aligned}
$$

Hence,

$$
\begin{aligned}
& b_{n}=\tilde{b}_{n}, \quad n \geq 2 ; \\
& c_{n}=\tilde{c}_{n}+\alpha_{n+1}-\alpha_{n}, \quad n \geq 2 \\
& \alpha_{n}\left(b_{n}-\tilde{b}_{n-2}\right)=0, \quad n \geq 3 \\
& \alpha_{n-1} c_{n}-\alpha_{n} \tilde{c}_{n-2}=0, \quad n \geq 3 \\
& \alpha_{2} b_{2}-\alpha_{2} \tilde{b}_{0}+b_{0} c_{2}-\tilde{b}_{0} c_{2}=0 \\
& b_{0}+b_{1}=\tilde{b}_{0}+\tilde{b}_{1} \\
& c_{1}=\tilde{c}_{1}+\alpha_{2}-\tilde{b}_{0} \tilde{b}_{1}+b_{0} b_{1} .
\end{aligned}
$$

Then, $\alpha_{n} \neq 0, n \geq 2$ by (4.24) and so $\tilde{b}_{n}=\tilde{b}_{n-2}, n \geq 3$ by (4.21) and (4.23). (4.16) and (4.17) follow from (4.22) and (4.24). Since $P_{1}(x)=Q_{1}(x)$, i.e., $b_{0}=\tilde{b}_{0}$, we have $b_{1}=\tilde{b}_{1}, b_{2}=\tilde{b}_{0}$, and $c_{1}=\tilde{c}_{1}+\alpha_{2}$ by (4.25), (4.26), and (4.27) from which we have $\tilde{b}_{2}=\tilde{b}_{0}$ and $\tilde{c}_{1}+\alpha_{2} \neq 0$. We also have, from (4.22) and (4.24),

$$
\alpha_{n}\left(1+\frac{\tilde{c}_{n-2}}{\alpha_{n-1}}\right)=\alpha_{n+1}\left(1+\frac{\tilde{c}_{n}}{\alpha_{n+1}}\right), \quad n \geq 3 .
$$

Thus

$$
\alpha_{n}\left(1+\frac{\tilde{c}_{n-1}}{\alpha_{n}}\right)\left(1+\frac{\tilde{c}_{n-2}}{\alpha_{n-1}}\right)=\mu(=\text { constant }), \quad n \geq 3 .
$$

Then, $\mu=0$ if and only if $\tilde{c}_{n}+\alpha_{n+1}=0$ for some $n \geq 1$. On the other hand, if $\tilde{c}_{n}+\alpha_{n+1}=0$ for some $n \geq 3$, then by (4.17), $\tilde{c}_{n-2}+\alpha_{n-1}=0$ so that either $\tilde{c}_{1}+\alpha_{2}=0$ or $\tilde{c}_{2}+\alpha_{3}=0$, both of which are impossible by (4.15) and (4.16). Hence, $\mu \neq 0$.

Conversely, assume that (ii) holds. Defi e $b_{n}, n \geq 0$ and $c_{n}, n \geq 1$ by (4.19). Then, (4.24) holds so that $c_{n} \neq 0, n \geq 1$. Now, it's easy to show that the three-term recurrence relation (4.2) holds. Hence, $\left\{P_{n}(x)\right\}_{n=0}^{\infty}$ is an MOPS. Finally assume that $\left\{P_{n}(x)\right\}_{n=0}^{\infty}$ is an MOPS relative to $\sigma$. Then we may assume (cf. (4.6)) that $\tau=\phi(x) \sigma, \phi(x)=x^{2}+\alpha x+\beta$. Then, it's easy to see that

$$
\phi(x) Q_{n}(x)=P_{n+2}(x)+A_{n} P_{n}(x), \quad n \geq 1,
$$


where $A_{n}=\frac{\left\langle\tau, Q_{n}^{2}\right\rangle}{\left\langle\sigma, P_{n}^{2}\right\rangle} \neq 0$. For $n \geq 2,(4.28)$ gives, by (4.14),

$$
\begin{aligned}
& \left(\tilde{b}_{n}+\tilde{b}_{n+1}+\alpha\right) Q_{n+1}(x)+\left(\tilde{c}_{n}+\tilde{c}_{n+1}+\tilde{b}_{n}^{2}+\alpha \tilde{b}_{n}+\beta-A_{n}+\alpha_{n+2}\right) Q_{n}(x) \\
& +\left(\tilde{b}_{n-1}+\tilde{b}_{n}+\alpha\right) \tilde{c}_{n} Q_{n-1}(x)+\left(\tilde{c}_{n-1} \tilde{c}_{n}+A_{n} \alpha_{n}\right) Q_{n-2}(x)=0
\end{aligned}
$$

so that

$$
\begin{gathered}
\tilde{b}_{n}+\tilde{b}_{n+1}+\alpha=\tilde{b}_{n-1}+\tilde{b}_{n}+\alpha=0, \quad n \geq 2 ; \\
\tilde{c}_{n}+\tilde{c}_{n+1}+\tilde{b}_{n}^{2}+\alpha \tilde{b}_{n}+\beta-A_{n}+\alpha_{n+2}=0, \quad n \geq 2 ; \\
\tilde{c}_{n-1} \tilde{c}_{n}+A_{n} \alpha_{n}=0, \quad n \geq 2 .
\end{gathered}
$$

Hence,

$$
\begin{aligned}
\alpha & =-\tilde{b}_{n}-\tilde{b}_{n+1}, \quad n \geq 0(\operatorname{cf} .(4.18)) \\
A_{n} & =-\frac{\tilde{c}_{n-1} \tilde{c}_{n}}{\alpha_{n}}, \quad n \geq 2 \\
\beta & =A_{n}-\alpha_{n+2}-\tilde{c}_{n}-\tilde{c}_{n+1}-\tilde{b}_{n}^{2}+\left(\tilde{b}_{n}+\tilde{b}_{n+1}\right) \tilde{b}_{n} \\
& =\tilde{b}_{n} \tilde{b}_{n+1}-\frac{c_{n-1} \tilde{c}_{n}}{\alpha_{n}}-\alpha_{n+2}-\tilde{c}_{n}-\tilde{c}_{n+1} \\
& =\tilde{b}_{n} \tilde{b}_{n+1}-\mu, \quad n \geq 2
\end{aligned}
$$

which gives (4.20). Finally, $\left\{P_{n}(x)\right\}_{n=0}^{\infty}$ is positive-defi ite if and only if $c_{n}>0$, $n \geq 1$ so that the conclusion concerning the positive-def niteness of $\left\{P_{n}(x)\right\}_{n=0}^{\infty}$ follows from (4.17) and (4.19).

Corollary 4.8. In Theorem 4.7, assume that $\tau$ is positive-definite and $\left\{P_{n}(x)\right\}_{n=0}^{\infty}$ is an MOPS. Then, $\left\{P_{n}(x)\right\}_{n=0}^{\infty}$ is a positive-definite MOPS if and only if

$$
\tilde{c}_{1}+\alpha_{2}>0, \quad \tilde{c}_{2}+\alpha_{3}-\alpha_{2}>0, \quad \text { and } \quad \alpha_{n-1} \alpha_{n}>0, \quad n \geq 3 \text {. }
$$

Corollary 4.9. Let $\left\{Q_{n}(x)\right\}_{n=0}^{\infty}$ be an MOPS relative to $\tau$ with real numbers $\tilde{c}_{n}$, $n \geq 1$, and define $\left\{P_{n}(x)\right\}_{n=0}^{\infty}$ by (4.14).

(i) If $\left\{Q_{n}(x)\right\}_{n=0}^{\infty}$ is a positive-definite MOPS and $\alpha_{n}, n \geq 2$, are real, then $P_{n}(x)$, $n \geq 3$, has at least $n-2$ nodal zeros (i.e., zeros of odd multiplicity) so that is at least $n-3$ simple zeros in $(\tilde{\xi}, \tilde{\eta})$.

(ii) If $\left\{P_{n}(x)\right\}_{n=0}^{\infty}$ is a positive-definite MOPS, then $Q_{n}(x)$ has at least $n-2$ nodal zeros so that is at least $n-3$ simple zeros in $(\xi, \eta)$.

Here, $[\xi, \eta]$ and $[\tilde{\xi}, \tilde{\eta}]$ are the true intervals of orthogonality for positive-definite $\operatorname{MOPSs}\left\{P_{n}(x)\right\}_{n=0}^{\infty}$ and $\left\{Q_{n}(x)\right\}_{n=0}^{\infty}$, respectively.

Proof. (i) When $\left\{Q_{n}(x)\right\}_{n=0}^{\infty}$ is a positive-defi ite MOPS and $\alpha_{n}, n \geq 2$, are real, $\left\{P_{n}(x)\right\}_{n=0}^{\infty}$ is a real PS. Let $x_{1}<x_{2}<\ldots<x_{k}$ be the nodal zeros of $P_{n}(x)$ in $(\tilde{\xi}, \tilde{\eta})$ and $\pi(x)=\prod_{j=1}^{k}\left(x-x_{j}\right)$. Then $\pi(x) P_{n}(x) \neq 0$ in $(\tilde{\xi}, \tilde{\eta})$ so that $\left\langle\tau, \pi(x) P_{n}(x)\right\rangle=\left\langle\tau, \pi(x)\left(Q_{n}(x)-Q_{n-2}(x)\right)\right\rangle \neq 0$. Hence, $k \geq n-2$. 
(ii) Assume that $\left\{P_{n}(x)\right\}_{n=0}^{\infty}$ is a positive-def nite MOPS. Then, by Theorem 4.7, $\alpha_{n}, n \geq 2$, are real since $\tilde{c}_{n}, n \geq 1$, are real. Hence, $A_{n}=-\frac{\tilde{c}_{n-1} \tilde{c}_{n}}{\alpha_{n}}, n \geq 2$, are also real so that $\phi(x) Q_{n}(x)=P_{n+2}(x)+A_{n} P_{n}(x)$ (cf. (4.28)) has at least $n$ nodal zeros in $(\xi, \eta)$. Hence, $Q_{n}(x)$ has at least $n-2$ nodal zeros in $(\xi, \eta)$.

As a converse to Theorem 4.7, we also have:

Theorem 4.10. Let $\left\{P_{n}(x)\right\}_{n=0}^{\infty}$ be an MOPS relative to $\sigma$ and define another monic $P S\left\{Q_{n}(x)\right\}_{n=0}^{\infty}$ recursively by (4.14). Then, the following are all equivalent:

(i) $\left\{Q_{n}(x)\right\}_{n=0}^{\infty}$ is also an MOPS (respectively, a positive-definite MOPS);

(ii) $\alpha_{n} \neq 0, n \geq 2 ; b_{n}=b_{n+2}, n \geq 0$; $\frac{\alpha_{n+1}}{\alpha_{n+2}} c_{n+2}=c_{n}-\alpha_{n+1}+\alpha_{n}, n \geq 1$

$\left(a_{1}=0\right)$ (respectively, $\frac{\alpha_{n+1}}{\alpha_{n+2}} c_{n+2}=c_{n}-\alpha_{n+1}+\alpha_{n}>0, n \geq 1$ ).

In this case, $\left\{Q_{n}(x)\right\}_{n=0}^{\infty}$ satisfy a three-term recurrence relation (4.3), where

$$
\tilde{b}_{n}=b_{n}, \quad n \geq 0 \quad \text { and } \quad \tilde{c}_{n}=\frac{\alpha_{n+1}}{\alpha_{n+2}} c_{n+2}, \quad n \geq 1,
$$

and is orthogonal with respect to $\tau=\left[x^{2}-\left(b_{0}+b_{1}\right) x+b_{0} b_{1}-\mu\right] \sigma$, where

$$
\mu=\alpha_{n}\left(1+\frac{c_{n}}{\alpha_{n}}\right)\left(1+\frac{c_{n+1}}{\alpha_{n+1}}\right) \neq 0, \quad n \geq 2 .
$$

Finally, we consider the following modifie problem: given an MOPS $\left\{Q_{n}(x)\right\}_{n=0}^{\infty}$, def ne a sequence of monic polynomials $P_{n}(x), n \geq t$, by

$$
P_{n}(x)=Q_{n}(x)-\alpha_{n} Q_{n-t}(x), \quad n \geq t,
$$

and ask: When can we complete $\left\{P_{n}(x)\right\}_{n=t}^{\infty}$ to an MOPS $\left\{P_{n}(x)\right\}_{n=0}^{\infty}$ by def ning $P_{0}(x)=1, P_{1}(x), \ldots, P_{t-1}(x)$. Here $t$ must be an integer $\geq 2$. First, we note that if there is an MOPS $\left\{P_{n}(x)\right\}_{n=0}^{\infty}$ relative to $\sigma$, then we still have (4.4), (4.5), and (4.6). We consider firs the case where $\alpha_{t}=0$.

Proposition 4.11. Let $\left\{Q_{n}(x)\right\}_{n=0}^{\infty}$ be an MOPS relative to $\tau$ and define $P_{n}(x)$, $n \geq t$, by (4.29), where $\alpha_{t}=0$. Then, $\left\{P_{n}(x)\right\}_{n=t}^{\infty}$ can be completed to an MOPS $\left\{P_{n}(x)\right\}_{n=0}^{\infty}$ if and only if $\alpha_{n}=0, n \geq t$ and $P_{n}(x)=Q_{n}(x), 0 \leq n \leq t-1$, that is, $\left\{P_{n}(x)\right\}_{n=0}^{\infty}=\left\{Q_{n}(x)\right\}_{n=0}^{\infty}$.

Proof. Assume that there is an MOPS $\left\{P_{n}(x)\right\}_{n=0}^{\infty}$ relative to $\sigma$. Then $\tau=\phi_{t}(x) \sigma$, $\operatorname{deg}\left(\phi_{t}\right) \leq t-1$ (cf. (4.5) and (4.6)). If $\phi_{t}(x) \equiv c$, a non-zero constant, then $\left\{Q_{n}(x)\right\}_{n=0}^{\infty}$ must be an MOPS relative to $\tau$ and $\sigma$ so that $\left\{P_{n}(x)\right\}_{n=0}^{\infty}=\left\{Q_{n}(x)\right\}_{n=0}^{\infty}$. If $\operatorname{deg} \phi_{t}=1$, then we may assume $\tau=(x-\mu) \sigma$ for some constant $\mu$ so that $\left\{Q_{n}(x)\right\}_{n=0}^{\infty}=\left\{P_{n}^{*}(\mu ; x)\right\}_{n=0}^{\infty}$, which is impossible by (2.5) and (4.29). Hence, $t \geq 3$. Then, (4.4) implies $\alpha_{n-1} c_{n}-\alpha_{n} \tilde{c}_{n-t}=0, n \geq t+1$ so that $\alpha_{n}=0$, $n \geq t$, that is, $P_{n}(x)=Q_{n}(x), n \geq t$. On the other hand, if $P_{n}(x)=Q_{n}(x)$ and $P_{n+1}(x)=Q_{n+1}(x)$, then

$$
\begin{aligned}
& \left(x-b_{n}\right) P_{n}(x)-c_{n} P_{n-1}(x)-P_{n+1}(x)=\left(x-b_{n}\right) Q_{n}(x)-c_{n} P_{n-1}(x)-Q_{n+1}(x) \\
& =\left(\tilde{b}_{n}-b_{n}\right) Q_{n}(x)+\tilde{c}_{n} Q_{n-1}(x)-c_{n} P_{n-1}(x)=0
\end{aligned}
$$

so that $P_{n-1}(x)=Q_{n-1}(x)$. Hence, we must have $P_{n}(x)=Q_{n}(x), n \geq 0$. The converse is trivial. 
Turning next to the case $\alpha_{t} \neq 0$, we consider the special case $t=2$. Then, as in Theorem 4.7, we can easily obtain:

Theorem 4.12. Let $\left\{Q_{n}(x)\right\}_{n=0}^{\infty}$ be an MOPS relative to $\tau$ and define $P_{n}(x), n \geq 2$ by (4.29) with $t=2$ and $\alpha_{2} \neq 0$. Then, $\left\{P_{n}(x)\right\}_{n=2}^{\infty}$ can be completed to an MOPS $\left\{P_{n}(x)\right\}_{n=0}^{\infty}$ if and only if $\alpha_{n} \neq 0, n \geq 2$ and

$$
\begin{gathered}
\tilde{b}_{n}=\tilde{b}_{n-2}, \quad n \geq 3 ; \\
\tilde{c}_{n}+\alpha_{n+1}-\alpha_{n}=\frac{\alpha_{n}}{\alpha_{n-1}} \tilde{c}_{n-2}, \quad n \geq 3 ; \\
\tilde{c}_{2}+\alpha_{3}-\alpha_{2} \neq 0 ; \\
\left(\tilde{c}_{2}+\alpha_{3}-\alpha_{2}\right)^{2}\left(\tilde{c}_{1}+\alpha_{2}-\tilde{b}_{0} \tilde{b}_{1}\right) \\
+\left(\tilde{b}_{0} \tilde{c}_{2}+\tilde{b}_{0} \alpha_{3}-\alpha_{2} \tilde{b}_{2}\right)\left\{\tilde{b}_{1}\left(\tilde{c}_{2}+\alpha_{3}-\alpha_{2}\right)+\alpha_{2} \tilde{b}_{2}-\tilde{b}_{0} \alpha_{2}\right\} \neq 0 .
\end{gathered}
$$

In this case, $\left\{P_{n}(x)\right\}_{n=0}^{\infty}$ satisfy the three-term recurrence relation (4.2), where

$$
b_{n}= \begin{cases}\frac{1}{c_{2}}\left(\tilde{b}_{0} \tilde{c}_{2}+\tilde{b}_{0} \alpha_{3}-\alpha_{2} \tilde{b}_{2}\right), & n=0 \\ \frac{1}{c_{2}}\left(\tilde{b}_{1} c_{2}+\alpha_{2} \tilde{b}_{2}-\alpha_{2} \tilde{b}_{0}\right), & n=1 \\ \tilde{b}_{n}, & n \geq 2,\end{cases}
$$

and

$c_{n}=\left\{\begin{array}{l}\tilde{c}_{1}+\alpha_{2}-\tilde{b}_{0} \tilde{b}_{1}+\frac{1}{c_{2}^{2}}\left(\tilde{b}_{0} \tilde{c}_{2}+\tilde{b}_{0} \alpha_{3}-\alpha_{2} \tilde{b}_{2}\right)\left(\tilde{b}_{1} c_{2}+\alpha_{2} \tilde{b}_{2}-\tilde{b}_{0} \alpha_{2}\right), \quad n=1 \\ \tilde{c}_{n}+\alpha_{n+1}-\alpha_{n}, \quad n \geq 2,\end{array}\right.$

and is orthogonal relative to $\sigma$ satisfying (4.20).

For $t>2$, we have:

Theorem 4.13. Let $\left\{Q_{n}(x)\right\}_{n=0}^{\infty}$ be an MOPS and define $P_{n}(x), n \geq t$, by (4.29) with $t \geq 3$ and $\alpha_{t} \neq 0$. If $\left\{P_{n}(x)\right\}_{n=t}^{\infty}$ can be completed to an MOPS $\left\{P_{n}(x)\right\}_{n=0}^{\infty}$, then:

$$
\begin{aligned}
\alpha_{n} \neq 0, \quad n \geq t \quad & \text { and } \quad \alpha_{n}=\alpha, \quad n \geq t+1 ; \\
\tilde{b}_{n} & =\tilde{b}_{n-t}, \quad n \geq t+1 ; \\
\tilde{c}_{n} & =\tilde{c}_{n-t}, \quad n \geq t+2 ; \\
\tilde{c}_{t+1} & =\frac{\alpha}{\alpha_{t+1}} \tilde{c}_{1} ;
\end{aligned}
$$

and $P_{t}(x)$ and $Q_{t-1}(x)+\frac{1}{\tilde{c}_{t}}\left(\alpha Q_{t}(x)-\alpha_{t} x+\alpha_{t} b_{t}\right)$ have no common zero. Conversely, if (4.30)-(4.33) hold and $Q_{t}(x)-\alpha_{t}$ and $Q_{t-1}(x)-\frac{1}{\tilde{c}_{t}}\left[\alpha Q_{1}(x)-\alpha_{t} x+\alpha_{t} b_{t}\right]$ have simple real interlacing zeros, then $\left\{P_{n}(x)\right\}_{n=t}^{\infty}$ can be completed to an MOPS $\left\{P_{n}(x)\right\}_{n=0}^{\infty}$. 
Proof. First assume that $\left\{P_{n}(x)\right\}_{n=t}^{\infty}$ can be completed to an MOPS $\left\{P_{n}(x)\right\}_{n=0}^{\infty}$. Then we have, from (4.2), for $n=t$,

$$
\left(\tilde{b}_{t}-b_{t}\right) Q_{t}(x)+\tilde{c}_{t} Q_{t-1}(x)-c_{t} P_{t-1}(x)+\alpha_{t+1} Q_{1}(x)-\alpha_{t} x+\alpha_{t} b_{t}=0 .
$$

Hence, together with (4.4), we have

$$
\begin{aligned}
\tilde{b}_{n}-b_{n} & =\tilde{c}_{n}-c_{n}, \quad n \geq t ; \\
\alpha_{n+1}-\alpha_{n} & =\alpha_{n} b_{n}-\alpha_{n} \tilde{b}_{n-t}=0, \quad n \geq t+1 ; \\
\alpha_{n-1} c_{n} & -\alpha_{n} \tilde{c}_{n-t}=0, \quad n \geq t+1 .
\end{aligned}
$$

Since $\alpha_{t} \neq 0, \alpha_{n} \neq 0$ for $n \geq t, \alpha_{n+1}=\alpha_{n}$ for $n \geq t+1$ and so $b_{n}=\tilde{b}_{n-t}$ for $n \geq t+1$ by (4.36) and (4.35). Hence, (4.30) and (4.31) hold. Then, (4.32) follows from (4.34) and (4.36) and (4.33) follows from (4.36) for $n=t+1$. Hence, we have

$$
P_{t-1}(x)=Q_{t-1}(x)+\frac{1}{\tilde{c}_{t}}\left(\alpha Q_{1}(x)-\alpha_{t} x+\alpha_{t} b_{t}\right)
$$

so that $P_{t}(x)$ and $P_{t-1}(x)$ cannot have a common zero. Conversely, assume that (4.30)-(4.33) hold and $P_{t}(x)$ and $Q_{t-1}(x)+\frac{1}{\tilde{c}_{t}}\left(\alpha Q_{1}(x)-\alpha_{t} x+\alpha_{t} b_{t}\right)$ have only simple real interlacing zeros. Defin $P_{t-1}(x)=Q_{t-1}(x)+\frac{1}{\tilde{c}_{t}}\left(\alpha Q_{1}(x)-\alpha_{t} x+\alpha_{t} b_{t}\right)$ and $b_{n}=\tilde{b}_{n}, c_{n}=\tilde{c}_{n}$ for $n \geq t$. Then, $c_{n} \neq 0, n \geq t$ and it's easy to show

$$
P_{n+1}(x)=\left(x-b_{n}\right) P_{n}(x)-c_{n} P_{n-1}(x), \quad n \geq t .
$$

Moreover, $P_{t}(x)$ and $P_{t-1}(x)$ must be real polynomials so that, by Wendroff's theorem [15], there are monic polynomials $\left\{P_{n}(x)\right\}_{n=0}^{t-2}$ such that

$$
P_{n+1}(x)=\left(x-b_{n}\right) P_{n}(x)-c_{n} P_{n-1}(x), \quad 0 \leq n \leq t-1, \quad \text { and } c_{n} \neq 0, \quad n \geq 1 .
$$

Hence, by Favard's theorem, $\left\{P_{n}(x)\right\}_{n=0}^{\infty}$ is an MOPS.

Acknowledgements. The f rst author (KHK) was partially supported by the BK-21 project and KOSEF(98-0701-03-01-5). The second author (DWL) was partially supported by BK-21 project. The third author (FM) was partially supported by Dirección General de Enseñanza Superior (DGES) of Spain under grant PB96-0120-C03-01. The fourth author (SBP) was partially supported by the Hwarangdae Research Institute.

\section{References}

1. Belmehdi, S.: On the left multiplication of a regular linear functional by a polynomial, in: Orthog. Polyn. and their Appl., Brezinski, C., Gori, L., Ronveaux, A., eds., 169-176, J.C. Baltzer AG, IMACS 1991

2. Branquinho, A., Marcellán, F.: Generating new classes of orthogonal polynomials. Internat. J. Math. \& Math. Sci. 19(4), 643-656 (1996)

3. Chihara, T.S.: On kernel polynomials and related systems. Boll. Un. Mat. Ital. 19(3), 451-459 (1964)

4. Chihara, T.S.: An introduction to orthogonal polynomials. New York: Gordon and Breach 1978 
5. Geronimus, Ya.L.: On polynomials orthogonal with respect to a given sequence of numbers. Zapiski Nanchno Issledovat'elno Inst. Math. Mekhaniki Kharkov St. U., 17 (1940) (Russian)

6. Iserles, A., Koch, P.K., Nørsett, S.P., Sanz-Serna, J.M.: On polynomials orthogonal with respect to certain Sobolev inner products. J. Approx. Th. 65(2), 151-175 (1991)

7. Krall, A.M.: Orthogonal polynomials satisfying fourth order differential equations. Proc. Royal Soc. Edinburgh 87(A), 271-288 (1981)

8. Krall, H.L.: On orthogonal polynomials satisfying a certain fourth order differential equation. Penn. St. College Studies No. 6, The Penn. St. Coll., Pa (1940)

9. Kwon, K.H., Lee, J.K., Yoo, B.H.: Characterizations of classical orthogonal polynomials. Results in Math. 24, 119-128 (1993)

10. Kwon, K.H., Park, S.B.: Two point masses perturbation of regular moment functionals. Indag. Math., N.S. 8, 1, 79-93 (1997)

11. Marcellán, F., Maroni, P.: Sur l'adjonction d'une masse de Dirac à une forme régulière et semi-classique. Ann. Mat. Pura ed Appl. CLXII, IV, 1-22 (1992)

12. Marcellán, F., Petronilho, J.: Orthogonal polynomials and coherent pairs: the classical case. Indag. Math., N.S. 6, 3, 287-307 (1995)

13. Maroni, P.: Variations around classical orthogonal polynomials, connected problems. J. Comp. Appl. Math. 48, 133-155 (1993)

14. Maroni, P.: Sur la suite de polynômes orthogonaux associée à la forme $u=\delta_{c}+$ $\lambda(x-c)^{-1} L$. Periodica Math. Hung. 21, 3, 223-248 (1990)

15. Wendroff, B.: On orthogonal polynomials. Proc. Amer. Math. Soc. 12, 554-555 (1961) 